\title{
TOEPLITZ OPERATORS ON $H^{2}$ SPACES
}

\author{
BY \\ ALLEN DEVINATZ( ${ }^{(1)}$
}

1. Let $L^{2}$ be the usual Lebesgue space with respect to Haar measure (normalized to one) on the unit circle and $H^{2}$ those elements of $L^{2}$ whose Fourier transforms vanish on the negative integers. The unitary map which takes any element of $L^{2}$ into its Fourier transform in $l^{2}$ (the space of square summable sequences on the integers) will take $H^{2}$ unitarily onto $l_{+}^{2}$ (the sequences of $t^{2}$ whose values vanish on the negative integers).

Let $\phi$ be a bounded measurable function on the circle group and $P$ the projection from $L^{2}$ onto $H^{2}$. By the Laurent operator $L_{\phi}$ on $L^{2}$ into itself we shall simply mean the operator defined by $L_{\phi} g=\phi g$. The Toeplitz operator $T_{\phi}$ shall be $P L_{\phi}$ restricted to $H^{2}$. The unitary Parseval operator from $L^{2}$ onto $l^{2}$ will induce corresponding Laurent and Toeplitz operators on $t^{2}$ and $t_{+}^{2}$ respectively which, of course, will be operators defined by convolution equations.

The space $H^{2}$ is clearly the closure in $L^{2}$ of the algebra $A$ consisting of continuous functions on the circle group whose Fourier coefficients vanish on the negative integers. The algebra $A$ is a prototype of what in the past few years has come to be called a Dirichlet algebra. As in the circle group case, a general Dirichlet algebra $A$ can be used to obtain $L^{2}$ and $H^{2}$ spaces and Laurent and Toeplitz operators can be defined. We are interested in the question under what circumstances a Toeplitz operator is invertible; i.e., is a one-one map of $H^{2}$ onto itself? We have solved this problem by showing that the problem of invertibility of a general Toeplitz operator can be reduced to the problem of invertibility of a special type of Toeplitz operator and that, in turn, this latter problem is equivalent to a problem solved by Helson and Szegö [6].

The invertibility problem on the transform space of $H^{2}$ of the circle group, i.e., the invertibility problem of Toeplitz operators on $l_{+}^{2}$, is of course the problem of solving a discrete Wiener-Hopf type convolution equation. Moreover, by a simple transformation the solution of the problem on $l_{+}^{2}$ leads to a solution of the usual $\mathrm{H}^{2}$ type of Wiener-Hopf equation on the real line. The problem of invertibility for $l_{+}^{2}$, also using the ideas of [6], was solved in $1960 \mathrm{by} \mathrm{H}$. Widom and announced by him, in part, in [13]. Without knowledge of Widom's work, we subsequently rediscovered his results and also found that the methods we had used would work

Received by the editors January 30, 1963.

(1) The research for this paper was partially supported by an NSF Senior Postdoctoral Fellowship and by grant NSF-G10715. 
for the general situation of Dirichlet algebras. We were also able to sharpen some of Widom's results for the circle group case and these will be presented in the last $\operatorname{section}\left({ }^{2}\right)$.

For historical accuracy, we should point out that for $\phi$ real the problem for $H^{2}$ of the circle group was completely solved by Hartman and Wintner [4]. That paper seems not to have been noticed by some of the more recent writers in this field.

2. Dirichlet algebras. In this section we shall state those theorems about Dirichlet algebras which we shall need for this paper. General references are $[7 ; 10 ; 11]$.

Let $X$ be a compact Hausdorff space and $A$ a closed subalgebra of $C(X)$, the space of complex continuous functions on $X$ with the usual supremum norm. $A$ is said to be a Dirichlet algebra if (a) $A$ separates points of $X$, (b) $1 \in A$, and (c) $\operatorname{Re} A$ is dense in $C_{R}(X)$, where $\operatorname{Re} A$ is the set of real parts of elements of $A$ and $C_{R}(X)$ is the algebra of real continuous functions on $X$.

Let $M$ be the maximal ideal space of $A$ and $0 \in M$. The Riesz representation theorem gives a unique bounded non-negative regular measure $d \mu$ on the Borel field of $X$ such that for any $f \in A$,

$$
\hat{f}(0)=\int_{X} f d \mu,
$$

where $\hat{f}$ is the representation of $f$ in $C(M)$.

If $1 \leqq p \leqq \infty, L^{p}$ shall designate the usual Lebesgue space with respect to $d \mu$ and for $1 \leqq p<\infty, H^{p}$ shall be the closure of $A$ in $L^{p} . H^{\infty}$ shall be the set of essentially bounded elements in $H^{1}$ with the norm taken as the essential supremum. It is a simple matter to check that $H^{\infty}$ is a Banach algebra.

Let $A_{0}$ be the (closed) subalgebra of $A$ consisting of those $f$ for which $\hat{f}(0)=0$. The space $H^{p}, 1 \leqq p \leqq \infty$, can be characterized as the collection of all elements in $L^{p}$ which are annihilated by every measure $f d \mu$ with $f \in A_{0}$. In other words, $g \in H^{p}$ if and only if $g \in L^{p}$ and $\int_{X} f g d \mu=0$ for every $f \in A_{0}$.

Several important theorems which are valid for the $H^{p}$ spaces of the circle group are also valid for the $H^{p}$ spaces generated by a Dirichlet algebra. A version of the Jensen formula, the factorization and minimum theorems of Szegö $[8 ; 9]$ and a closure theorem of Beurling [1] are valid in the general situation. We shall list these below and refer to [7] for the proofs.

THEOREM A. If $f \in H^{1}$, then

$$
\log |\hat{f}(0)| \leqq \int_{X} \log |f| d \mu .
$$

(2) In a correspondence with Professor Widom, in July 1962, he also informed me that he had lectured on his work in 1960 at the Institute for Advanced Study. I am grateful to Professor Widom for freely discussing his unpublished results with me. 
If $f \in H^{1}$ we shall say $f$ is an outer factor if and only if $\hat{f}(0)>0$ and

$$
\log \hat{f}(0)=\int_{X} \log |f| d \mu
$$

THEOREM B. Let $w$ be a non-negative function on $X$ summable with respect to $d \mu$. A necessary and sufficient condition for $w$ to have a unique representation

$$
w(x)=|f(x)|^{2}
$$

a.e.-d $\mu$ on $X$, with $f$ an outer factor in $H^{2}$, is that

$$
\int_{X} \log w d \mu>-\infty
$$

TheOREM C. Let $w$ be as in Theorem B; then

$$
\exp \int_{X} \log w d \mu=\inf _{\boldsymbol{g}} \int_{X}|1+g|^{2} w d \mu,
$$

where $g$ ranges over elements of $A_{0}$. The left side is to be considered zero if $\int_{X} \log w d \mu=-\infty$.

THEOREM D. If $f \in H_{1}, \int \log |f| d \mu>-\infty$, then we may write

$$
f=g h \text { a.e. }-d \mu,
$$

where $h$ is an outer factor in $H^{1}$ and $g \in H^{\infty}$ with $|g(x)|=1$ a.e.-d $\mu$. The decomposition is unique. Further $h=k^{2}, k$ an outer factor in $H^{2}$.

We shall say that an element $g \in H^{\infty}$ is an inner factor if and only if a.e. $|g(x)|=1$.

THEOREM E. If $f, 1 / f \in H^{1}$, then $f$ differs from an outer factor by $a$ multiplicative constant of unit modulus. Conversely, if $f$ is an outer factor and $1 / f \in L^{1}$, then $1 / f \in H^{1}$.

A result of Beurling [1] on invariant subspaces is also valid in the general situation. A subspace $S \subseteq H^{p}$ is said to be invariant if and only if $g S \subseteq S$ for every $g \in A$.

THEOREM F. Let $f \in H^{2}$ and $S_{f}$ the smallest closed invariant subspace containing $f$. Then $S_{f}=H^{2}$ if and only iff is, up to a multiplicative constant of unit modulus, an outer factor.

From this result and the fact that outer factors in $H^{1}$ may be factored into products of elements in $H^{2}$ one may easily prove a corresponding result for $H^{1}$.

3. The conjugation operation. Let $X, A, M, d \mu$, be as in the previous section. If $u \in \operatorname{Re} A$, then there exists a $v \in \operatorname{Re} A$ such that $u+i v \in A$. Since the constants are in $A$ we can choose $v$ so that $\int v d \mu=0$. Such a function $v$ is unique a.e. $-d \mu$. 
Indeed, suppose $v_{1}$ is another such function; i.e., $u+i v_{1} \in A$ and $\int v_{1} d \mu=0$. It follows that $v-v_{1} \in A \cap \operatorname{Re} A$. But the only real functions in $A$ differ from constants a.e. This together with the normalization of $v$ and $v_{1}$ shows that they are equal a.e.

If $u \in \operatorname{Re} A$ and $v \in \operatorname{Re} A$ such that $u+i v \in A$ and $\int v d \mu=0$, let us designate by $C u$ the equivalence class of measurable functions which differ from $v$ only a.e. Call $C u$ the conjugate of $u$ (more precisely, the conjugate of the equivalence class of $u$ ) and $C$ the conjugation operator. It is clear that $C$ is a linear transformation acting on the real linear space $\operatorname{Re} A$ and by linearity it can be extended to the complex linear space $\operatorname{Re} A+i \operatorname{Re} A$.

By means of a beautifully simple construction S. Bochner [15] has shown that the Riesz conjugate function theorem is valid for $C$. That is to say, if $1<p<\infty$ then $C$ is a bounded operator in the norm of $L^{p}$. Using the regularity of $d \mu$, this means that $C$ can be extended, as a bounded linear operator to act on $L^{p}$ into itself. In this context, the Riesz conjugate function theorem is as follows:

THEOREM G. If $1<p<\infty$ there exists a unique continuous linear operator, $C_{p}$, taking $L^{p}$ into itself, such that for every $f \in L^{p}, \int C_{p} f d \mu=0$, and if $u \in \operatorname{Re} A$ there exists $a \tilde{u}$ in the equivalence class $C_{p} u$ such that $u+i \tilde{u} \in A$.

We should note that it is immediate that if $1<r, s<\infty$ and $f \in L^{r} \cap L^{s}$, then $C_{r} f=C_{s} f$. Hence if $f \in L^{p}$ for all $1<p<\infty$ we will drop the subscript on the conjugation operator and simply write $C f$.

The proof of the following theorem follows the same line of reasoning as in the circle group case. See [14, p. 257].

THEOREM H. If $u$ is real and measurable, and $|u| \leqq 1$, then

$$
\int \exp (\lambda|C u|) d \mu \leqq A_{\lambda}<\infty
$$

for $0 \leqq \lambda<\pi / 2$.

Proof. Suppose $g=u+i \tilde{u}$, where $u \in \operatorname{Re} A, \tilde{u} \in C u$ and $|u| \leqq 1$. By expanding $\exp \pm i \lambda g$ into a power series and using the linear and multiplicative properties of the ideal $0 \in M$, or by use of the Lévy-Wiener theorem, we arrive at the fact that $\int \exp \pm i \lambda g d \mu=\exp \pm i \lambda \hat{g}(0)=\exp \pm i \lambda \hat{u}(0)$, where $\hat{u}(0)=\int u d \mu$. Taking real parts we get

$$
\int \exp \pm \lambda C u \cos \lambda u d \mu=\cos \lambda \hat{u}(0) \leqq 1
$$

Since $\exp \lambda|C u| \leqq \exp \lambda C u+\exp -\lambda C u$ and $\cos \lambda u \geqq \cos \lambda$ for $|u| \leqq 1$ and $0 \leqq \lambda<\pi / 2$, it follows that

$$
\int \exp \lambda|C u| d \mu \leqq 2 / \cos \lambda
$$


Suppose now that $u$ is real and measurable and $|u| \leqq 1$. Since $d \mu$ is regular we may find a sequence $\left\{u_{n}\right\} \subset \operatorname{Re} A$ such that $\left|u_{n}\right| \leqq 1$ and $u_{n} \rightarrow u$ almost everywhere. Then by Fatou's lemma

$$
\int \exp \lambda|C u| d \mu \leqq \liminf _{n \rightarrow \infty} \int \exp \lambda\left|C u_{n}\right| d \mu \leqq 2 / \cos \lambda .
$$

COROLlaRY. If $u$ is real and measurable and $|u|<\pi / 2-\varepsilon$ for $\varepsilon>0$, then there exists a $\delta>0$ such that if $1 \leqq p<1+\delta, \exp (i u-C u) \in H^{p}$.

Proof. By the previous theorem it is clear that there exists a $\delta>0$ so that if $0 \leqq p<1+\delta$, then $\exp p|C u| \in L^{1}$. Hence, if we can show that for every $g \in A$ with $\hat{g}(0)=0$,

$$
\int g \exp (i u-C u) d \mu=0
$$

then we are done.

There exists a sequence $\left\{u_{n}\right\} \subseteq \operatorname{Re} A$ such that $\left|u_{n}\right|<\pi / 2-\varepsilon$ and $u_{n} \rightarrow u$ a.e. Since $\left\{\exp C u_{n}\right\}$ is uniformly bounded in $L^{p}, 1 \leqq p<1+\delta$, it follows that for any fixed $p$ in this range there exists a subsequence $\left\{v_{n}\right\}$ of $\left\{u_{n}\right\}$ so that $\exp \left(i v_{n}-C v_{n}\right)$ $\rightarrow \exp (i u-C u)$ weakly in $L^{p}$. There is a $\tilde{v}_{n}$ in the equivalence class $C v_{n}$ so that $v_{n}+i \tilde{v}_{n} \in A$, and hence $\exp \left(i v_{n}-\tilde{v}_{n}\right) \in A$. If $g \in A$ with $\hat{g}(0)=0$,

$$
0=\int g \exp \left(i v_{n}-C v_{n}\right) d \mu \rightarrow \int g \exp (i u-C u) d \mu .
$$

4. The problem of Helson-Szegö. All of the definitions and results of this section are due to Helson and Szegö [6]. Let $R$ and $S$ be manifolds in a Hilbert space $H$. Let

$$
\rho=\sup \{|(f \mid g)|, f \in R, g \in S,\|f\|=\|g\|=1\} .
$$

It is clear that $0 \leqq \rho \leqq 1$. If $\rho<1$, the manifolds are said to be at a positive angle. It is an easy matter to check that $0 \leqq \rho<1$ if and only if there exists a $0 \leqq \sigma<1$ such that for every $f \in R, g \in S$,

$$
2(1-\sigma)\|f\|\|g\| \leqq\|f+g\|^{2} .
$$

Suppose now that $w$ is a real non-negative measurable function in $L^{1}$. Let $L^{2}(w)$ be the usual Lebesgue space with respect to the measure $w d \mu$. Let $R=H^{2}(w)$ and $S=\bar{H}_{0}^{2}(w)$, where $H^{2}(w)$ is the subspace of $L^{2}(w)$ generated by $A$ and $\bar{H}_{0}^{2}(w)$ is the subspace of $L^{2}(w)$ generated by $\bar{A}_{0}$, where $\bar{A}_{0}$ is the set of functions which are complex conjugates of those $g \in A$ such that $\hat{g}(0)=0$.

Let $P$ be the projection of $L^{2}$ on $H^{2}$; i.e.,

$$
P u=\frac{1}{2}[u+\hat{u}(0)+i C u]
$$


where $\hat{u}(0)$ is defined by (2.1). Using the formula (4.2), it is easy to verify that $R$ and $S$ are at a positive angle if and only if $P$ is a bounded operator in $L^{2}(w)$; i.e., $R$ and $S$ are at a positive angle if and only if there exists a $B>0$ such that

$$
\|P u\|_{w} \leqq B\|u\|_{w} .
$$

Notice that from (4.3) it is almost immediate that $C$ is bounded in $L^{2}(w)$ if and only if $P$ is bounded in $L^{2}(w)$.

If we set $P u=f,(I-P) u=g$ then it follows from (4.4) that a necessary and sufficient condition that $H^{2}(w)$ and $\bar{H}_{0}^{2}(w)$ are at a positive angle is that there exists a positive constant $B$ so that either one of the two following inequalities holds for every $f \in H^{2}(w)$ and $g \in \bar{H}_{0}^{2}(w)$ :

$$
\|f\|_{w} \leqq B\|f+g\|_{w},\|g\|_{w} \leqq B\|f+g\|_{w} .
$$

If $\int \log w d \mu>-\infty$, then by Theorem C there exists an outer factor $h \in H^{1}$ such that $w=|h|$. Under these conditions and taking $-\pi \leqq \operatorname{Arg} z<\pi$ we have

THEOREM I. In order that $H^{2}(w)$ and $\bar{H}_{0}^{2}(w)$ be at a positive angle it is necessary and sufficient that there exist an $\varepsilon>0$ and a $g \in H^{\infty}$ so that $1 / g \in H^{\infty}$ and $\mid$ Arg $g h \mid<\pi / 2-\varepsilon$.

The proof follows the line of proof of Lemma 1 on p. 126 of [6]. However, several points of the proof are somewhat different, especially the fact $\left({ }^{3}\right)$ that $1 / g \in H^{\infty}$. Complete details will appear in [16].

5. The invertibility of Toeplitz operators. Our object in this section is to give necessary and sufficient conditions that a Toeplitz operator be invertible. We shall first do this for real kernels obtaining a generalization of a theorem of Hartman and Wintner [4]. Our notations are as in the previous sections.

We first prove the following which generalizes Theorems (ii) and (iv) of $[4$, p. 870$]$.

LEMMA 1. Let $f$ be a real valued measurable function on $X$. Necessary and sufficient conditions that the equation

$$
f(x) g(x)=1+h(x)=k(x)
$$

have solutions $g$ and $h$ in $H^{2}$ with $\hat{h}(0)=0$ are:

(i) $\operatorname{sgn} f$ is constant, a.e.,

(ii) $f$ and $1 / f$ are in $L^{1}$.

Proof. Sufficiency. From (i) we may suppose without any loss of generality that $f \geqq 0$. From (ii) we get from the Szegö factorization theorem (Theorem B)

(3) This was pointed out to me by my colleague I. I. Hirschman, Jr. and his students Frank Cholewinski and Jeffrey Davis in their seminar on Toeplitz forms. I am grateful to them for this, as well as for several other comments which materially improved some parts of the exposition. 
$f=|k|^{2}, k$ an outer factor in $H^{2}$. Since $1 / f \in L^{1}$ it follows that $1 / k \in L^{2}$ and hence by Theorem $E$ it follows that $1 / k \in H^{2}$. Hence, multiplying through by a suitable constant if necessary, this gives equation (5.1).

Necessity. Multiply equation (5.1) by $k$ to give

$$
f(x) g(x) k(x)=|k(x)|^{2}=f_{1}(x) .
$$

Since $\hat{k}(0)=1$, it follows from Theorem A that $\int \log f_{1} d \mu \geqq 0$ and hence $f_{1}(x) \neq 0$ a.e. $-d \mu$. Since $g, k \in H^{2}$ it follows that $g k \in H^{1}$. From the fact that $f$ and $f_{1}$ are different from zero a.e. and real it follows that $g k$ is real and hence must be constant a.e. This already proves (i) since $f_{1}>0$ a.e.

The fact that $g(x) k(x)=c$ a.e. gives $g(x)=c k(x)^{-1}$ and hence $k^{-1} \in L^{2}$, and consequently $1 / f=g k^{-1} \in L^{1}$. Also, by the same reasoning, $f=k g \in L^{1}$ which gives (ii).

Recall now that we define $L_{\phi} g=\phi g$ and for $g \in H^{2}, T_{\phi} g=P L_{\phi} g$, where $P$ is the projection from $L^{2}$ onto $H^{2}$. Designate the spectrum of the Toeplitz operator $T$ by $\sigma\left(T_{\phi}\right)$. The following theorem is due to Hartman and Wintner [4] in the circle group case.

THEOREM 1. If $\phi$ is real, bounded and measurable, then $\sigma\left(T_{\phi}\right)=$ [ess. inf $\phi$, ess. $\sup \phi]$.

Proof. Since $T_{\phi}$ is self-adjoint the spectrum must be real. Suppose ess. $\inf \phi<\lambda<$ ess. $\sup \phi$ and $T_{\phi}-\lambda I$ has a bounded inverse. Then for any $h \in H^{2}$ there exists a $g \in H^{2}$ such that $\left(T_{\phi}-\lambda I\right) g=h$. Choose $h \equiv 1$; then $(\phi(x)-\lambda) g(x)=1+k(x), k \in H^{2}, \hat{k}(0)=0$. By the previous lemma $\phi(x)-\lambda$ must have constant sign, which is a contradiction. This shows that $\sigma\left(T_{\phi}\right)$ $\supseteq$ [ess. inf $\phi$, ess. $\sup \phi]$.

Now, suppose $\lambda \notin[$ ess. inf $\phi$, ess. $\sup \phi]$, then $\phi(x)-\lambda$ has constant sign, $|\phi(x)-\lambda| \geqq \delta>0$ and hence by Theorem $\mathrm{B}$, we have

$$
\phi_{\lambda}=\phi-\lambda= \pm \Psi \psi, \psi \in H^{2},|\psi| \geqq \delta .
$$

Hence, $T_{\phi_{\lambda}}= \pm T_{\bar{\psi}} T_{\psi}$. But $T_{\psi}$ has a bounded inverse, namely $T_{1 / \psi}$ and hence so does $T_{\bar{\psi}}=T_{\psi}^{*}$. Consequently, $T_{\phi_{\lambda}}$ has a bounded inverse showing that $\sigma\left(T_{\phi}\right) \subseteq[$ ess. inf $\phi$, ess. $\sup \phi]$.

We shall now shift our attention to a complex valued function $\phi$ and give necessary conditions that $T_{\phi}$ be invertible.

Lemma 2. Let $\phi$ be a bounded measurable function on $X$. Necessary conditions that $T_{\phi}$ is invertible are:

(1) ess. $\inf |\phi|>0$,

(2) $T_{\phi /|\phi|}$ is invertible,

(3) $\phi /|\phi|=\Psi \mid \psi$, where $\psi, 1 / \psi \in H^{2}$. 
Proof. The assumption is that $T_{\phi}$ has an inverse. Hence, there exists a unique $g \in H^{2}$ such that $T_{\phi} g=1$. In other words, we have $\phi(x) g(x)=h(x)=1+k(x)$ where $k \in H^{2}$ and $\hat{k}(0)=0$. Therefore, $\log |\phi|+\log |g|=\log |h|$ and since by Theorem $\mathrm{A}, \log |h|$ belongs to $L^{1}$, it follows that $\log |\phi|$ belongs to $L^{1}$. Indeed, since $\int \log { }^{+}|g| \leqq \int|g|<\infty$, if $\log |g| \notin L^{1}$ we must have $\int \log |g|=-\infty$ and hence $\int \log |\phi|=\infty$ which is impossible. It follows from Theorem B that we may write $|\phi|=\bar{\chi} \chi$, where $\chi$ is an outer factor in $H^{\infty}$.

If we set $\theta(x)=\phi(x) /|\phi(x)|$ then we may write

$$
T_{\phi}=T_{\bar{\chi}} T_{\theta} T_{\chi} .
$$

Since $\chi$ is an outer factor, by Theorem $\mathrm{F}$ the collection of elements of the form $T_{\chi} p(=\chi p)$ for $p \in A$ is dense in $H^{2}$. By hypothesis, $T_{\chi}$ has a bounded inverse, namely $T_{\phi}^{-1} T_{\bar{\chi}} T_{\theta}$. Hence it follows that the range of $T_{\chi}$ is closed and consequently all of $H^{2}$.

The fact that $T_{\chi}$ is invertible implies $T_{\chi}^{*}=T_{\bar{\chi}}$ is invertible and therefore $T_{|\phi|}=T_{\bar{\chi}} T_{\chi}$ is invertible. Theorem 1 implies that ess. inf $|\phi|>0$ which is condition (1).

The fact that $T_{\chi}, T_{\bar{\chi}}$ and $T_{\phi}$ are invertible implies $T_{\theta}$ and $T_{\theta}^{*}=T_{\bar{\theta}}$ are invertible which is condition (2). There exist unique $g, g_{1} \in H^{2}$ such that $T_{\theta} g=1$ and $T_{\theta} g_{1}=1$; i.e., $\theta(x) g(x)=h(x)=1+\bar{z}(x)$ and $\bar{\theta}(x) g_{1}(x)=h_{1}(x)=1+\bar{z}_{1}(x)$, where $z, z_{1} \in H^{2}$ with $\hat{z}(0)=\hat{z}_{1}(0)=0$. It follows that $g g_{1}=\overline{h h}_{1}$ and hence $g g_{1}$ and $h h_{1}$ are a.e. constant. Therefore, by Theorem $\mathrm{E}$ all of these functions and their inverses are, up to multiplicative constants, outer factors. Also note, that from the fact that $1 / \theta=\bar{\theta}$, that $\theta(x) h(x)=\bar{g}(x)$ and hence from the (1-1) character of $T_{\theta}, h(x) /(\hat{g})^{-}(0)=g(x)$. This gives (3).

Our object now is to prove the equivalence of the problem of Helson-Szegö with the problem of invertibility of a certain class of Toeplitz operators.

TheOREM 2. Let $\theta=\psi / \psi$, where $\psi$ and $1 / \psi$ are in $H^{2}$, and set $w=|\psi|^{2}$. $A$ necessary and sufficient condition that $T_{\theta}$ be invertible is that

$$
\rho=\sup \left\{\left|(f \mid g)_{w}\right| ; f \in H^{2}(w), g \in \bar{H}_{0}^{2}(w)\|f\|=\|g\|=1\right\}<1 .
$$

Proof. The range of $T_{\theta}$ is dense in $H^{2}$. Indeed, $\left(T_{\theta} g \mid h\right)=\int g h \psi / \psi d \mu=0$ for $h \in H^{2}$ and all $g \in A$ implies $h \psi / \bar{\psi} \in\left(H^{2}\right)^{\perp}$ which in turn implies $h \psi$ is constant. Since $1 / \psi$ is outer, $h \psi=0$ and hence $h=0$.

Now, suppose $\rho<1$; according to the discussion of $\S 4$, there exists a $B>0$ such that

$$
\|f\|_{w} \leqq B\|f+g\|_{w}, \quad f \in H^{2}(w), \quad g \in \bar{H}_{0}^{2}(w) .
$$

Since $1 / \psi \in H^{2}$ it follows that every $h \in H^{2}$ is of the form $h=f \psi$ where $f=h / \psi \in H^{1}$, and every $k \in\left(H^{2}\right)^{\perp}$, is of the form $g \bar{\psi}$, where $\bar{g} \in H^{1}, \hat{g}(0)=0$. 
Since $\psi$ is an outer factor, from Theorem $\mathrm{F}$ we can find $\left\{f_{n}\right\} \subseteq A, f_{n} \psi \rightarrow h$ in $H^{2}$. Hence,

$$
\int\left|f_{n}-f\right|^{2} w d \mu=\int\left|f_{n} \psi-h\right|^{2} d \mu \rightarrow 0
$$

and therefore $f \in H^{2}(w)$. In the same way we can see that $g \in \bar{H}_{0}^{2}(w)$.Consequently,

$$
\|g+f\|_{w}=\|g \bar{\psi}+f \bar{\psi}\|_{2}=\left\|g \bar{\psi}+L_{\theta}(f \psi)\right\|_{2},
$$

where $L_{\theta}$ is the Laurent operator given by $L_{\theta} k(x)=6(x) k(x)$. Since $L_{\theta}(f \psi)-T_{\theta}(f \psi) \in\left(H^{2}\right)^{\perp}$ it follows from the above discussion that we may find $g \in \bar{H}_{0}^{2}(w)$ such that

$$
L_{\theta}(f \psi)=T_{\theta}(f \psi)-g \bar{\psi}
$$

Using this $g$ in (5.4), from (5.3) and (5.5), we get

$$
\|f\|_{w}=\|f \psi\|_{2} \leqq B\left\|T_{\theta}(f \psi)\right\|_{2} .
$$

Since every element of $H^{2}$ is of the form $f \psi$ with $f \in H^{2}(w)$ it follows that $T_{\theta}$ has a bounded inverse on $H^{2}$.

Conversely, suppose $T_{\theta}$ has a bounded inverse on $H^{2}$. We may write

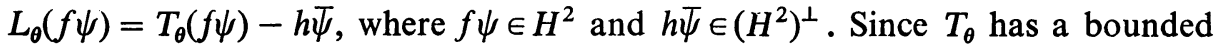
inverse, there exists a $B>0$ such that

$$
\|f\|_{w}=\|f \psi\|_{2} \leqq B\left\|h \bar{\psi}+L_{\theta}(f \psi)\right\|_{2}=B\left\|T_{\theta}(f \psi)\right\|_{2} .
$$

But since $-h \bar{\psi}$ is the projection of $L_{\theta}(f \psi)$ onto $\left(H^{2}\right)^{\perp}$ we have

$$
\left\|h \bar{\psi}+L_{\theta}(f \psi)\right\|_{2} \leqq\left\|g \bar{\psi}+L_{\theta}(f \psi)\right\|_{2}=\|g \bar{\psi}+f \bar{\psi}\|_{2}=\|g+f\|_{w}
$$

for any $g \bar{\psi} \in\left(H^{2}\right)^{\perp}$. Hence (5.7) and (5.8) imply

$$
\|f\|_{w} \leqq B\|g+f\|_{w} .
$$

If $f \in H^{2}(w)$ then $f \psi \in H^{2}$, and if $g \in \bar{H}_{0}^{2}(w), g \bar{\psi} \in\left(H^{2}\right)^{\perp}$. Hence (5.9) holds for all $f \in H^{2}(w)$ and $g \in \bar{H}_{0}^{2}(w)$. This, taken together with the results of $\$ 4$, concludes the proof of the theorem $\left({ }^{4}\right)$.

Let us now cast this result in a form which will lead to necessary and sufficient conditions on a function $\phi$ in order that $T_{\phi}$ be invertible.

LEMma 3. Suppose $\phi$ is a bounded measurable function on $X$ which is different from zero a.e. The operator $T_{\phi|| \phi \mid}$ is invertible if and only if there exists a $g \in H^{\infty}$ such that $1 / g \in H^{\infty}$ and an $\varepsilon>0$ such that $|\operatorname{Arg}(g \phi)| \leqq \pi / 2-\varepsilon$.

(4) We use the fact that $1 / \psi \in H^{2}$ only in the sufficiency part of the proof. If $X$ is the circle group and $\psi$ an outer factor in $H^{2}$ and $\rho<1$, then Theorem 1 of [6] implies $1 / \psi \in H^{2}$. In this case, then, the problem of invertibility of $T_{\theta}$ and the problem of Helson-Szegö are equivalent in the sense that we do not have to assume $a$ priori that $1 / w \in L^{1}$. We have shown in [16] that this is also true for a general Dirichlet algebra. 
Proof. Let $v=\operatorname{Arg}(g \phi)$ and

$$
G=e^{-c v+i v} .
$$

It follows from the corollary to Theorem $H$ that $G$ and $1 / G$ are in $H^{p}$ for some $p>1$. If we set

$$
F=g^{-1} G
$$

it follows that for the same $p, F$ and $1 / F$ are in $H^{p}$. Now

$$
F /|F|=e^{i \operatorname{Arg} F}=e^{i \operatorname{Arg} g^{-1} G}=e^{i \operatorname{Arg} g^{-1}} e^{i \operatorname{Arg} G} .
$$

But $e^{i \operatorname{Arg} G}=e^{i \operatorname{Arg} g} \cdot e^{i \operatorname{Arg} \phi}$, and hence

$$
F /|F|=e^{i \operatorname{Arg} \phi}=\phi /|\phi| \text {. }
$$

Since $F$ and $1 / F$ are in $H^{p}$ with $p>1$, it follows that $F$ is an outer factor. It follows from Theorem $\mathrm{D}$ that $F=K^{2}, K$ an outer factor in $H^{2}$.

Take $\psi=1 / K$; then $\psi$ and $1 / \psi$ are in $H^{q}, q>2$ and

$$
\bar{\psi} / \psi=\frac{|\psi|^{2}}{\psi^{2}}=e^{-i \operatorname{Arg} \psi^{2}}=e^{i \operatorname{Arg} F}=\phi /|\phi|
$$

Further, $\left|\operatorname{Arg}\left(g^{-1} \psi^{2}\right)\right|=|\operatorname{Arg} G|=|\operatorname{Arg}(g \phi)| \leqq \pi / 2-\varepsilon$. Hence using Theorem I and our previous theorem, we see that $T_{\phi /|\phi|}$ is invertible.

Conversely, suppose $T_{\phi /|\phi|}$ has a bounded inverse; then by Lemma 2 , $\phi /|\phi|=\psi / \psi, \psi, 1 / \psi \in H^{2}$ and by Theorem 2 and Theorem I there exists a $g \in H^{\infty}$ such that $1 / g \in H^{\infty}$ and an $\varepsilon>0$ such that $\left|\operatorname{Arg}\left(g^{-1} \psi^{2}\right)\right| \leqq \pi / 2-\varepsilon$. It follows that

$$
g \phi /|g \phi|=\frac{\left|g^{-1} \psi^{2}\right|}{g^{-1} \psi^{2}}=e^{-i \operatorname{Arg} g^{-1} \psi^{2}}=e^{i \operatorname{Arg} g \phi} .
$$

Hence $|\operatorname{Arg} g \phi| \leqq \pi / 2-\varepsilon$.

THEOREM 3. Suppose $\phi$ is a bounded measurable function on $X$. Necessary and sufficient conditions that $T_{\phi}$ is invertible are:

(1) ess. inf $|\phi|>0$.

(2) There exists a $g \in H^{\infty}$ such that $1 / g \in H^{\infty}$ and an $\varepsilon>0$ such that $\mid$ Arg $g \phi \mid \leqq \pi / 2-\varepsilon$.

Proof. The necessity follows from Lemmas 2 and 3.

The sufficiency follows from formula (5.2) of Lemma 2. Indeed, from (1) we may write $|\phi|=\bar{\chi} \chi$, where $\chi$ and $1 / \chi$ are in $H^{\infty}$. Therefore, as the proof of Lemma 2 shows, $T_{\phi}$ is inverible if and only if $T_{\phi /|\phi|}$ is invertible, and the invertibility of this latter operator follows from (2) and Lemma 3.

Let us state this theorem in an equivalent but slightly different form. 
COROLlaRY. If $\phi$ is a bounded measurable function, then $T_{\phi}$ is invertible if and only if

$$
\phi=g e^{u+i v}
$$

where $g, 1 / g \in H^{\infty}, u$ and $v$ are real with $u \in L^{\infty}$ and $\|v\|_{\infty}<\pi / 2$.

Proof. If $\phi$ is of the given form, then setting $h=e^{u+i v}$ we see that ess. inf $|h|>0$ and $\|\operatorname{Arg} h\|_{\infty}<\pi / 2$. Hence, $T_{h}$ is invertible by Theorem 3. Further

$$
T_{\phi}=T_{h} T_{g},
$$

and since $T_{g}$ is invertible, with inverse $T_{1 / g}$, it follows that $T_{\phi}$ is invertible.

To prove the necessity, suppose $T_{\phi}$ is invertible. According to Theorem 3 there is a $g \in H^{\infty}$ such that $1 / g \in H^{\infty}$ and $\left\|\operatorname{Arg} g^{-1} \phi\right\|_{\infty}<\pi / 2$. Hence,

$$
\phi=g e^{\log \left|g^{-1} \phi\right|+i \operatorname{Arg} g^{-1} \phi} .
$$

Since ess. inf $|\phi|>0, w=\log \left|g^{-1} \phi\right| \in L^{\infty}$ and also $v=\operatorname{Arg} g^{-1} \phi$ has the desired property.

Corollary $\left({ }^{5}\right)$. If $X$ is the circle group, A the algebra of continuous functions on $X$ with Fourier coefficients vanishing on the negative integers and 0 the origin of coordinates, then $T_{\phi}$ is invertible if and only if

$$
\phi=\gamma e^{u+i(v+C w)}
$$

where $u, v$, and $w$ are real, $u, w \in L^{\infty},\|v\|_{\infty}<\pi / 2$, and $\gamma$ is constant with $|\gamma|=1$.

Proof. If $\phi$ is of this form, set $h=e^{u-w+i v}$ and $g=\gamma e^{w+i C w}$. Then $g, 1 / g \in H^{\infty}$ and $\phi$ has the form of the previous corollary.

Conversely if $T_{\phi}$ is invertible, using the previous corollary and the fact that every outer factor in $H^{\infty}$ of the circle group is of the form $g=\exp (w+i C w+i \alpha)$, where $w$ is real and in $L^{\infty}$ and $\alpha$ is a real constant, gives the result.

COROLLARY. The spectrum of $T_{\phi}$ is contained in the closed convex hull of the essential range of $\phi$.

Proof. If $\lambda$ is not in the closed convex hull of the essential range of $\phi$, then it is clear that there exists an $\alpha$ such that $\left\|\operatorname{Arg}\left\{e^{i \alpha}(\phi-\lambda)\right\}\right\|_{\infty}<\pi / 2$.

In general the spectrum of $T_{\phi}$ does not fill out the entire closed convex hull of the essential range of $\phi$, as the reader may verify by any number of simple counterexamples.

6. Some results for the circle group. In this section we shall assume that the compact space $X$ is the circle group, the Dirichlet algebra $A$ is the collection

(5) This is the theorem obtained by Widom for the circle group. After this paper was written we discovered it was valid for a general Dirichlet algebra by showing that any outer factor has the form given in the necessity part of the proof. Details of this latter fact will appear in [16]. 
of continuous functions on $X$ whose Fourier coefficients vanish on the negative integers and the maximal ideal 0 is the origin of coordinates of the complex plane. It is easily checked that the measure $d \mu$ is the Haar measure of $X$ normalized to one.

THEOREM 4. Suppose $\phi$ is a measurable function on $X$ and $\theta(x)$ a determination of the argument of $\phi(x)$ which is continuous except for jump discontinuities of magnitude $\leqq \pi$, except possibly at one point (where the jump discontinuity may have magnitude $>\pi)\left({ }^{6}\right)$. Then $T_{\phi /|\phi|}$ is invertible if and only if all jump discontinuities have magnitude $<\pi\left({ }^{7}\right)$.

Proof. (a) Sufficiency. It may be easily shown [3, p. 832] that given $\varepsilon>0$ there exists a set $\left\{I_{k}\right\}_{1}^{n}$ of mutually disjoint open arcs in the circle $X$ such that $X-\bigcup I_{k}$ is a finite set of points and

$$
\sup _{x, y \in I_{k}}|\theta(x)-\theta(y)|<\varepsilon .
$$

This means there exists a $\delta>0$ such that the magnitude of the jumps of $\theta(x)$ are $<\pi-\delta$. If we take $\varepsilon=\delta / 2$, it is clearly possible to find a continuously differentiable function $\psi(x)$ such that $|\theta(x)-\psi(x)|<\pi / 2-\varepsilon$.

Let us now proceed as in [6]. Let

$$
g=e^{c \psi-i \psi}
$$

It is clear that $g, 1 / g \in H^{\infty}$ and

$$
\frac{g \phi}{|g \phi| 1}=e^{i \operatorname{Arg} g \phi}=e^{-i \psi+i \theta}
$$

Hence $|\operatorname{Arg} g \phi|<\pi / 2-\varepsilon$ and the sufficiency follows from Theorem 3 .

(b) Necessity. Let us set $J(y)=y$ for $0 \leqq y<2 \pi$ and then extend $J$ periodically to the whole axis. Suppose the jump discontinuities of $\theta$ are at $\left\{e^{i y_{k}}\right\}$, where the index $k$ has been arranged so that the magnitude of the jumps are nonincreasing. Choose weights $\alpha_{k}$ and $n$ so large that upon setting

$$
\phi_{n}\left(e^{i y}\right)=\phi\left(e^{i y}\right) \exp \left(i \sum_{1}^{n} \alpha_{k} J\left(y-y_{k}\right)\right)
$$

a determination of the argument of $\phi_{n}$ can be chosen so as to be continuous with the possible exception of jump discontinuities of magnitude less than a given

(6) The restriction on the magnitudes of the jumps of $\theta(x)$ is not an essential one since, if there exists a determination of the argument of $\phi$ which has only jump discontinuities, then there exists a determination of the argument as in the theorem.

(7) This generalizes Theorem IV of Widom [12] to the situation where the argument may have a countable number of jumps. It also generalize Theorem 6 of Helson-Szegö [6]. 
$\varepsilon>0$. Part (a) of this proof shows that $T_{\phi_{n} /\left|\phi_{n}\right|}$ is invertible and hence we can write $\phi_{n} /\left|\phi_{n}\right|=\bar{\chi}_{n} \mid \chi_{n}$. Indeed, from the proof of Lemma 3 and Theorem $\mathrm{H}$, it follows that $\chi_{n}, 1 / \chi_{n} \in H^{q}$, where $q \rightarrow \infty$ as $\varepsilon \rightarrow 0$. Since $T_{\phi /|\phi|}$ is, by hypothesis, invertible, we may write $\phi /|\phi|=\bar{\chi} \mid \chi$, where $\chi, 1 / \chi \in H^{p}$ for some $p>2$.

Let us suppose (without loss of generality) that $y_{1}=0$ and that the magnitude of all other jumps are $\leqq \pi$. Let $\alpha_{1}=m+\beta$, where $m$ is an integer and $|\beta| \leqq 1 / 2$.

$$
\frac{\bar{\chi}_{n}}{\chi_{n}}=e^{i m y} \frac{\bar{\chi}}{\chi} \frac{\bar{\eta}_{n}}{\eta_{n}}
$$

where

$$
1 / \eta_{n}\left(e^{i y}\right)=\gamma_{n}\left(1-e^{i y}\right)^{\beta} \prod_{2}^{n}\left(1-e^{i\left(y-y_{k}\right)}\right)^{\alpha_{k}},
$$

$\left|\alpha_{k}\right| \leqq 1 / 2, \gamma_{n}$ is constant with $\left|\gamma_{n}\right|=1$, and $-\pi / 2<\arg \left(1-e^{i t}\right) \leqq \pi / 2$. It is clear that $\eta_{n}$ and $1 / \eta_{n}$ are in $H^{p}$ for $1 \leqq p<2$ and therefore $\chi \eta_{n}$ and $1 / \chi \eta_{n}$ are in $H^{p}$ for some $p>1$. Therefore, by making $n$ sufficiently large, $\chi_{n} / \chi \eta_{n} \in H^{1}$. But from (6.1)

$$
\bar{\chi}_{n} / \bar{\chi} \bar{\eta}_{n}=e^{i m y} \chi_{n} / \chi \eta,
$$

and this is possible only if $m \leqq 0$. On the other hand making $n$ sufficiently large, $\chi \eta_{n} / \chi_{n} \in H^{1}$ and from (6.1) we get

$$
\chi \eta_{n} / \chi_{n}=e^{i m y} \bar{\chi} \bar{\eta}_{n} / \bar{\chi}_{n}
$$

and this can be true only if $m \geqq 0$. Hence we must have $m=0$ and from (6.2) we have $\chi_{n} / \chi \eta_{n} \equiv C_{n}$ for $n$ sufficiently large, where $C_{n}$ is a constant.

Now, $\chi$ and $1 / \chi \in H^{p}$ for some $p>2$ and given any $1 \leqq q<\infty$, if $n$ is sufficiently large, $\chi_{n}, 1 / \chi_{n} \in H^{q}$. Hence, if $n$ is sufficiently large, $\chi_{n} / \chi$ and $\chi / \chi_{n}$ are in $H^{2}$ which means $\eta_{n}$ and $1 / \eta_{n}$ are in $H^{2}$. Hence all jumps must have magnitude $<\pi$. This concludes the proof of the theorem.

Since this paper was written, H. Widom [17] has proved that the spectrum of a Toeplitz operator defined on the $H^{2}$ space of this section has a connected spectrum. Using results of [16] his arguments extend to the Dirichlet algebra situation.

\section{REFERENCES}

1. A. Beurling, On two problems concerning linear transformation in Hilbert space, Acta Math. 81 (1949), 239-255.

2. A. Calderón, F. Spitzer and H. Widom, Inversion of Toeplitz matrices, Illinois J. Math. 3 (1959), 490-498.

3. A. Devinatz and I. I. Hirschman, Jr., The spectra of muitiplier transforms on $l^{p}$, Amer. J. Math. 80 (1958), 829-842.

4. P. Hartman and A. Wintner, The spectra of Toeplitz's matrices, Amer. J. Math. 76 (1954), 867-882.

5. H. Helson and D. Lowdenslager, Prediction theory and Fourier series in several variables Acta Math. 99 (1958), 165-202. 
6. H. Helson and G. Szegö, A problem in prediction theory, Ann. Mat. Pura Appl. 51 (1960). 107-138.

7. K. Hoffman, Analytic functions and logmodular Banach algebras, Acta Math. 108 (1962), 271-317.

8. G. Szegö, Beiträge zur Theorie der Toeplitzschen Formen. I, II, Math. Z. 6(1920), 167-202; ibid. 9 (1921), 167-190.

9. - Ueber die Randwerte einer analytischen Funktion, Math. Ann. 84 (1921), 232-244.

10. J. Wermer, Dirichlet algebras, Duke Math. J. 27 (1960), 373-381.

11. - Banach algebra and analytic functions, Advances in Math. 1 (1961), 51-102.

12. H. Widom, Inversion of Toeplitz matrices. II, Illinois J. Math. 4 (1960), 88-99.

13. - Inversion of Toeplitz matrices. III, Abstract 564-246, Amer. Math. Soc. Notices 7 (1960), 63.

14. A. Zygmund, Trigonometric series, Vol. I, Cambridge Univ. Press, Cambridge, 1959.

15. S. Bochner, Generalized conjugate and analytic functions without expansions, Proc. Nat. Acad. Sci. USA 45 (1959)., 855-857.

16. A. Devinatz, Conjugate function theorems in Diricheet algebras, Rev. Un. Mat. Argentina (to appear).

17. H. Widom, On the spectrum of a Toeplitz operator (to appear).

WASHINGTON UNIVERSITY,

ST. Louis. MissourI 